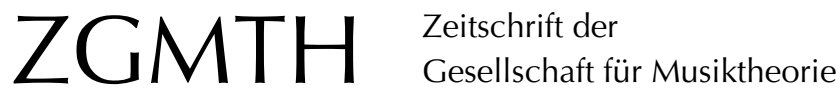

Breyer, Knud (2009): Peter H. Smith, Expressive Forms in Brahms's Instrumental Music: Structure and Meaning in His Werther Quartet, Bloomington: Indiana University Press 2005. ZGMTH 6/1, 173-176. https://doi.org/10.31751/425

(C) 2009 Knud Breyer

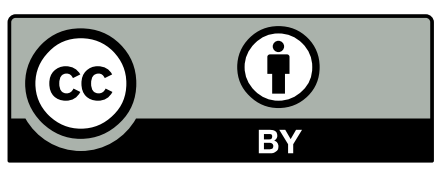

Dieser Text erscheint im Open Access und ist lizenziert unter einer Creative Commons Namensnennung 4.0 International Lizenz.

This is an open access article licensed under a Creative Commons Attribution 4.0 International License.

veröffentlicht / first published: 15/02/2009

zuletzt geändert / last updated: 14/10/2010 


\section{Peter H. Smith, Expressive Forms in Brahms's Instrumental Music: Structure and Meaning in His Werther Quartet, Bloomington: Indiana University Press 2005}

Mit seiner Unterschrift unter das sogenannte 'Manifest gegen die Neudeutschen ' hatte Johannes Brahms 1860 seine Gegnerschaft zur Neudeutschen Schule bekundet. Während jedoch die Partei der Neudeutschen insofern eine homogene Gruppe bildete, als sie sich auf das Dogma berief, in der Gegenwart sei musikalischer Fortschritt nur im Rahmen neuer Gattungen - Symphonische Dichtung und Musikdrama - und auf der Grundlage eines außermusikalischen Vorwurfs möglich, war die Gegenpartei zersplittert. Ihr Spektrum reichte von den traditionalistischen Akademikern, für die die Musikgeschichte seit Haydn und Mozart eine des Verfalls klassischer Normen war, bis zu Brahms, der vor der Veröffentlichung der Serenaden op. 11 und op. 16 noch als Romantiker im Geiste Schumanns rezipiert und von den Akademikern bekämpft wurde.

Eine ähnliche Konstellation, wenngleich unter genau umgekehrten Vorzeichen, kennzeichnet die aktuelle musikwissenschaftliche Diskussion im angloamerikanischen Raum. Der traditionalistischen Musiktheorie, die ihre Aufgabe primär in der immanenten Analyse musikalischer Strukturen sieht, steht eine heterogene, gemeinhein unter das Schlagwort der 'New Musicology subsumierte Gruppierung gegenüber, die das musikalische Kunstwerk nicht als autonomen Gegenstand, sondern prädeterminiertes Ergebnis einer Widerspiegelung sei es allgemein-gesellschaftlicher, sei es persönlich-biographischer Strukturen ansieht.

Zwischen den Extrempositionen von Autonomie- und Heteronomieästhetik nehmen Johannes Brahms als Komponist und Peter $\mathrm{H}$. Smith als Theoretiker eine vergleichbare Mittelposition ein. Ob sich Brahms mit seiner Unterstützung des `Manifests` auch gegen die Programmmusik an sich wandte - und nicht primär gegen den Alleinvertretungsanspruch der Neudeutschen in Bezug auf den musikalischen Fortschritt - ist überaus fraglich. Zwar wirkt Brahms' CEuvre auf den ersten Blick wie das auskomponierte Gegenkonzept zu den Neudeutschen: Die Symphonische Dichtung wird ausgespart, die Fortschrittsfähigkeit der klassischen Gattungen bewiesen und handwerkliches Ethos selbst dort gegen das Primat des poetischen Zusammenhangs gesetzt, wo dieses, wie im Falle des Klavierstücks, zu den Erwartungen an die Gattung gehört. Andererseits aber hatte Brahms gegenüber Clara Schumann das Unternehmen Eduard Hanslicks, Musik gegen die seiner Meinung nach „verrottete Gefühlsästhetik" auf die Darstellung "tönend bewegter Formen« zu reduzieren, gleichfalls zurückgewiesen (»Sein Buch ,Vom Musikalisch Schönen ... wollte ich lesen, fand aber gleich beim Durchsehen so viel Dummes, daß ich's ließ.»). ${ }^{1}$ Auf vergleichbare Weise stellt sich Smith einerseits gegen eine allzu naive Ineinssetzung von biographischem Ich, intelligiblem Ich und Kunstwerk, misst andererseits aber intrinsischen Merkmalen der musikalischen Struktur gleichfalls eine extrinsische Bedeutung bei.

Im Mittelpunkt von Smiths Untersuchung Expressive Forms in Brahms's Instrumental Music steht das Klavierquartett c-Moll op. 60. Der Gegenstand ist mit Bedacht gewählt. Das Klavierquartett gehört-neben dem langsamen Satz aus der Klaviersonate op. 5 und dem Intermezzo op. $117 \mathrm{Nr} .1$ (beiden Kompositionen stellt Brahms Gedichtzeilen voran) - zu den wenigen Beispielen, in denen Brahms selbst auf einen programmatischen Vorwurf ver-

1 Zit. nach Knepler, Georg (1997), „Brahms historische und ästhetische Bedeutung", in: Johannes Brahms oder die Relativierung der sabsoluten Musik, hg. von Hanns-Werner Heister, Hamburg: von Bockel, 36-48, hier: 41. 
wiesen hat. Anders als bei op. 5 und op. 117 Nr. 1 fehlt dieser Hinweis im veröffentlichten Notentext des Klavierquartetts; er verbleibt stattdessen im intimen Bereich der privaten Korrespondenz. Die Verständnishilfen, die Brahms drei Adressaten zu verschiedenen Zeiten gab, änderten allmählich ihre Konnotation. Während er Hermann Deiters 1868 noch schreibt, dass er sich in Bezug auf den ersten Satz ganz allgemein einen Mann vorzustellen habe, "der sich eben totschießen will, und dem nichts anderes mehr übrig bleibt", bittet er Theodor Billroth 1874 bereits, sich »als Kuriosum - etwa eine Illustration zum letzten Kapitel vom Mann im blauen Frack und gelber Weste« vorzustellen. Er verbindet also die allgemeine Selbstmordthematik mit einer konkreten literarischen Anspielung auf Goethes Werther. Diese literarische Figur bezieht er schließlich in einem Brief an Fritz Simrock 1875 auf sich selbst. Mit sarkastischer Ironie verspricht er seinem Verleger, zur Komplettierung dieser Illustration eine Fotografie von sich selbst zu schicken.

Damit ergeben sich bei op. 60 vier Stränge der Verbindung von - wie es bei Smith im Untertitel heißt - "structure and meaning", die der Autor abwägen und schließlich zusammenführen möchte. Da Brahms selbst auf eine Bezeichnung wie 'Werther-Quartetts verzichtete, ist op. 60 zunächst als ein Stück absoluter Musik anzusehen und auf seine immanente Struktur hin zu untersuchen. Aufgrund der Deiters-Briefstelle wäre dann zu fragen, inwieweit die strukturellen Sachverhalte Ausdrucksqualitäten implizieren, die ins Verhältnis zur ausweglosen Verzweiflung eines Selbstmörders zu bringen sind. Drittens wäre nach einer möglichen intertextuellen Relation zwischen dem Quartett und dem Roman zu suchen und schließlich diese auf einen möglichen biographischen Hintergrund bei Brahms hin zu befragen.

Letzterer wird durch die frühen Entstehungszeitpunkte des ersten Satzes sowie eines Adagios, das jedoch nichts mit dem späteren langsamen Satz von op. 60 zu tun hat, nahegelegt. Die Ursprünge des Quartetts reichen in das Jahr 1855 zurück - eine für Brahms emotional bewegte Zeit. Seine Liebe zu Clara Schumann musste aus Gründen sowohl der gesellschaftlichen Konvention als auch der persönlichen Integrität unerfüllt bleiben. Zwar verweist Smith zu Recht darauf, dass die Dreieckskonstellation im Hause Schumann nicht mit jener im Werther-Roman zu vergleichen ist, da Brahms als Gast in den bestehenden Haushalt eines verheirateten Ehepaares zog (im Roman dagegen stellt sich die verhängnisvolle Konstellation erst durch das Eintreffen des Verlobten Albert ein). Ferner ist es Robert Schumann, der einen Selbstmordversuch unternahm und daraufhin das Heim verlassen musste - was an der Unerfüllbarkeit von Brahms' Liebe vermutlich nichts änderte. Smith lässt aber erstens völlig außer Acht, dass Brahms in den 50er Jahren die Identifikation mit Roman(anti)helden liebte (insbesondere mit E.T. A. Hoffmanns Kapellmeister Kreisler) und dass Werther für ganze Generationen die Symbolfigur adoleszenter Liebesleidender bildete. Zweitens geht er nicht näher auf einen Brief an Simrock ein, in dem Brahms sogar noch Jahrzehnte später die explizite Verbindung zwischen sich selbst und Werther zieht. Obwohl Smith grundsätzliche Bedenken gegen die biographische Interpretation hat, will er sie nicht unversucht lassen, wählt hierfür aber merkwürdigerweise das erst später (1869 oder sogar erst 1874) hinzugefügte Andante. Sein Ansatz ist hier so schlicht, und das analytische Niveau fällt so deutlich gegenüber dem Übrigen ab, dass man Smiths Vorbehalte, die biographische Interpretation sei eine zweifelhafte Methode, sofort teilen möchte. Smith personalisiert die (unterschiedlichen Klangoder Charaktersphären zuzuordnenden) thematischen Bereiche und ordnet sie den drei Protagonisten Robert, Clara und Johannes zu. Was diesen Ansatz interessant gemacht hätte, nämlich eine genaue kontrapunktische Analyse, die die Kommunikationsstruktur zwischen dem von Smith als paternalistisch bezeichneten ,Robert-Thema im Cello, dem delikaten ‘Claras-Thema und dem synkopierenden sohannesı-Thema zutage gefördert hätte, bleibt Smith leider schuldig. Sie hätte den prima-vista Eindruck näher beleuchten 
können, dass das synkopierende Thema die dialogische Harmonie der 'Ehepaars-Themen nachhaltig stört, was bei einer biographischen Interpretation als ein Ausdruck von Schuldgefühl gedeutet werden könnte.

In eine vertiefte biographische Deutung will Smith aber ebenso wenig einsteigen wie in eine literarisch-programmatische. Letztere weist er mit dem etwas trivialen Argument zurück, dass in op. 60 keine konkreten Handlungsmomente des Romans naturalistischabbildend geschildert würden (z. B. »Werther pulls the trigger" [283]). Stattdessen geht er von jenem hermeneutischen Fingerzeig im Deiters-Brief aus, der viel unbestimmter und aus einer auktorialen Position heraus auf die Selbstmordthematik zu sprechen kommt. Damit spielt er eher auf die emotionale Qualität dieser psychischen Grenzerfahrung im Allgemeinen an, eine Erfahrung, die mit Gefühlen existentieller Angst, Verzweiflung und innerer Zerrüttung verbunden ist. Bei dem Versuch, diese extreme Gefühlssphäre als besonderen Inhalt nicht nur des ersten Satzes, sondern des gesamten Quartetts auszuweisen, greift Smith auf eine mehrstufige Argumentation zurück. Da diese, wenngleich aus verschiedenen Perspektiven, letztlich dann doch um einen relativ überschaubaren Bestand an Befunden kreist, kommt es zu einer gewissen Redundanz und Umständlichkeit in der Beweisführung. Entschädigt wird der Leser dafür durch eine ebenso präzise wie vielfältige, systematische wie historische Aspekte beleuchtende Untersuchung auf hohem analytischen Niveau.

Obgleich sich Smith ausdrücklich auf Robert S. Hattens Methode der formalisierten Gegenüberstellung semantischer Oppositionstypen beruft, ließe sich seine Argumentation ebensogut auf die barocke musikalisch-rhetorische Figuren- und Toposlehre zurückführen. Sie geht gleichfalls von der Isomorphie emotionaler und musikalischer Strukturen aus und weist insbesondere Abweichungen von der Norm als emblematische Bedeutungsträger aus. Zwar entdeckt Smith auch in op. 60 noch unmittelbare Ableger dieser Tradition wie Seufzermotivik, an den passus duriusculus` erinnernde chromatische Abgänge oder pausendurchsetzte Vorhaltbildungen, also traditionelle Figuren, die Leid und Verzagen darstellen. Ferner lassen sich die unheimlich wirkenden leiterfremden und als Pizzicato gespielten Töne e (T. 28ff.), aber auch leere Quintklänge in der Tat als Entfremdungssymbol deuten. Vor allem aber überträgt Smith die Idee, Normabweichungen semantisch zu deuten, originell auf die Formanalyse. Hierbei geht es ihm weniger um die individuelle Abweichung von einem Formschema, als vielmehr darum, innerhalb individueller Formverläufe das aufzuspüren, was mit Leonard B. Meyer als snoncongruencer bezeichnet werden kann. Um sich eine sichere empirische Basis zu schaffen, bezieht Smith eine ganze Reihe von Kompositionen sowohl von Brahms als auch von Haydn, Mozart, Beethoven und Schubert in seine Überlegungen mit ein. Ausgehend von der Vermutung, Brahms habe op. 60 ganz bewusst nachträglich von cisnach c-Moll transponiert, werden vor allem Werke in der stragischen، Tonart c-Moll herausgehoben behandelt. Besonderes Augenmerk legt Smith hierbei auf die Alt-Rhapsodie op. 53, das Schicksalslied op. 54, Schuberts Quartettsatz D 701 und Haydns Symphonie in c-Moll Nr. 95, wobei es im Falle von op. 53 und op. 54 insbesondere um intertextuelle Korrespondenzen zu op. 60 geht, die offenbaren, dass Brahms hier wie dort zu ähnlichen Ausdrucksmitteln greift. Den Vergleich mit dem Quartettsatz nutzt Smith auch, um gegen James Websters Auffassung zu argumentieren, der junge Brahms habe sich vor allem an Schubert orientiert. Im Rahmen der angesprochenen c-Moll-Werke - 5. Symphonie von Beethoven, 1. Symphonie, Schicksalslied und Alt-Rhapsodie von Brahms - ist op. 60 die einzige Komposition, die keine per aspera ad astra-Lösung anbietet. Deshalb aber, wie Smith, von einem dezidiert tragischen Ende des Quartetts zu sprechen, ist diskussionswürdig. Donald F. Tovey hatte auf op. 60 die Folie der klassischen Tragödie angewendet. Smith widerspricht diesem Ansatz insbesondere mit dem Hinweis auf den szusammenbrechenden Choral im Finale, der nicht - wie in den Vergleichswerken 1. Symphonie und Mendels- 
sohns c-Moll-Trio - als positiver und triumphaler Erlösungstopos dient. Auf der anderen Seite aber kann das beiläufige C-Dur-Ende in op. 60 durchaus als lapidare Wiederherstellung einer vom Drama des untergegangenen Helden unabhängigen und unbeeindruckten Ordnung interpretiert werden.

Wer den Selbstmörder als jemanden versteht, der einen radikalen Bruch mit sich selbst als Subjekt und mit seiner Umwelt vollzieht, muss - um auf Smiths Kernanliegen zurückzukommen - seine "strategic interpretation" (Hatten) darauf ausrichten, eine entsprechende Diskontinuität in der musikalischen Struktur nachzuweisen. Smith verschränkt hierzu drei prominente Analyseverfahren, die die Beschreibung organischer Einheit erlauben und somit auch umgekehrt als Prüfstein für deren Gefährdung taugen. In erster Linie bezieht sich Smith - ausgehend von Milton Babbitts Begriff des dimensionalen Kontrapunkts - auf die Entfaltung harmonischer Dispositionen. Eher am Rande knüpft er an Schönbergs Befund an, musikalische Ökonomie bei Brahms verdanke sich der motivischen Entfaltung einer rudimentären motivischen Substanz. Schließlich wird dem Bottom-up-Verfahren Schönbergs das Top-down-Verfahren der Stimmführungsanalyse Schenkers zu Seite gestellt.

Folglich werden von Smith vor allem formale und harmonische Aspekte inhaltlich - und das heißt hier: als Dokumente des Scheiterns - gedeutet. Für den ersten Satz konstatiert Smith, harmonische Spannungen entlüden sich nicht positiv, sondern würden vielmehr verschluckt. Der Seitensatzkomplex, in der traditionellen Gegentonart Es-Dur stehend, hebt zwar hoffnungsvoll an, durch die Variationen, deren letzte dann auch wieder in das düstere Hauptthema zurückleitet, wirken, so Smith, die positiven Gefühle aber eher manisch konstruiert als natürlich empfunden. Die Durchführung lässt diesen Eindruck Gewissheit werden. Nachdem das Grandioso des Hauptsatzes durch die entfernte Tonart H-Dur als bloß fantasierte Überwindung erscheint, zeigt sich auch der Seitensatz als falsches Versprechen. Er ist krisenhaft umgeprägt und erscheint in G-Dur, also der notwendig ins tragische c-Moll zurückfallenden Dominante. Die Reprise ist in op. 60 neu komponiert. Ihre Klimax ist ein verzweifelter Aufschrei (T. 117), dem der totale Zusammenbruch folgt.

Wie gesagt ist nicht sicher, ob Brahms die Selbstmordthematik nur auf den ersten Satz bezogen hat. Smith aber meint, auch insofern in den anderen Sätzen eine gewisse Kontinuität erkennen zu können, als beispielsweise das Scherzo keinen Entspannungspol bildet, sondern ebenfalls angespannt bleibt und auch krisenhafte Ausbrüche kennt. Das Andante wiederum zeigt die Gegenwelt nur als entfernten Traum in E-Dur, also jener Tonart, die in den 'Entfremdungspizzicati< des ersten Satzes bereits anklang. Ferner könnte hier der Solosonatenton als Symbol für die Vereinzelung gedeutet werden. Das Thema des Finales ist semantisch doppelt konnotiert. Es lehnt sich an die Lieder Regenlied und Nachklang op. 59 Nr. 3 und 4 an, in denen Schönheit und Vergänglichkeit der Jugend thematisiert werden. Ferner wird der deshalb nur gebrochen hoffnungsvolle Gestus wieder zurückgenommen, zum einen durch das c-Moll und seine Dominante, zum anderen durch das Zerschellen des Chorals, der ohnehin von vornherein durch die sfalscher Begleitung angreifbar schien.

Smith geht es offenbar vor allem darum, gegen die von ihm als theorieskeptisch dargestellte `New Musicology` zu zeigen, dass und vor allem wie traditionelle Verfahren der Formanalyse auch für die semantische Forschung fruchtbar gemacht werden können. Dies ist ihm einerseits überzeugend gelungen. Andererseits fällt sein Analysenetz relativ klein und grobmaschig aus, obwohl es aus vielen Fäden zusammen gewoben wurde: Die verschiedenen Ansätze haben erstens jeweils nur eine begrenzte Tragweite und werden zweitens von Smith nicht konsequent durchdekliniert. Hier hätte man sich statt der weitläufigen und nicht immer ohne polemische Verkürzung geführten Methodendiskussion eine im Detail gründlichere, vollständige und zusätzlich auch stärker an Oberflächenphänomenen orientierte Arbeit gewünscht.

Knud Breyer 